\title{
Estado Nutricional e Consumo Alimentar de Gestantes em uma Maternidade do Município de São Paulo
}

\author{
Nutritional Status and Food Consumption of Pregnant Women in a Maternity \\ Hospital in the City of São Paulo
}

\author{
Rouglana Ribeiro Barbosa ${ }^{1}$ \\ Marcela Maria Pandolfi
}

\section{RESUMO}

Objetivo: Descrever o estado nutricional e o consumo alimentar de pacientes internadas na Casa da Gestante de um Hospital e Maternidade no Município de São Paulo. Metodologia: Trata-se de estudo transversal, descritivo, realizado com 54 gestantes. $O$ estado nutricional pré-gestacional foi avaliado usando o Índice de Massa Corporal (IMC) preconizado pela World Health Organization (WHO) e o gestacional utilizado a curva de Atalah. Os marcadores de consumo alimentar foram avaliados por meio de formulário do Ministério da Saúde. Para as associações estatísticas, utilizou-se o teste do Qui-quadrado de Pearson e o teste exato de Fisher, considerando estatisticamente significante valor de $p \leq 0,05$. Resultados: Ocorreu maior prevalência de obesidade tanto no período pré-gestacional $(41,4 \%)$ quanto no gestacional $(50 \%)$ e de ganho ponderal excessivo $(49,05 \%)$ e insuficiente $(39,6 \%)$. Observou-se associação entre eutrofia no período pré-gestacional $(p=0,019)$ e gestacional $(p=0,036)$, com ganho de peso adequado. A obesidade mostrou-se associada ao ganho excessivo $(p=0,000)$. Verificou-se associação entre o diabetes gestacional e a obesidade $(p=0,013)$, assim como entre a hipertensão arterial crônica e a obesidade $(p=0,020)$. A maioria referiu o uso de equipamentos eletrônicos, simultaneamente, à realização das refeições e ter consumido alimentos in natura no dia anterior à entrevista, enquanto, o consumo de alimentos ultraprocessados variou entre $20 \%$ e $37 \%$. No entanto, $68,5 \%$ referiram ter consumido bebidas adoçadas. Conclusão: O presente estudo encontrou maior prevalência de obesidade no período pré-gestacional e gestacional, além de expressivo percentual do consumo de alimentos in natura e menor frequência do consumo de ultraprocessados.

\section{DESCRITORES}

Estado nutricional. Consumo Alimentar. Gestantes. Comportamento Alimentar. Guias Alimentares.

\begin{abstract}
Objective: To describe the nutritional status and food consumption of pregnant women at a Maternity Hospital in the city of São Paulo. Methodology: This was a cross-sectional, descriptive study, carried out with 54 pregnant women. Nutritional status before pregnancy was assessed using the Body Mass Index (BMI) as recommended by the World Health Organization (WHO), and for nutritional status during pregnancy, the Atalah curve was used. Food consumption markers were applied using a Ministry of Health form. Pearson's chi-square test and Fisher's exact test were used for statistical associations, considering a statistically significant value of $p \leq 0.05$. Results: There was a higher prevalence of obesity both before pregnancy $(41.4 \%)$ and during pregnancy $(50 \%)$, in addition to excessive weight gain $(49.05 \%)$ followed by insufficient weight gain (39.6\%). An association was observed between eutrophy before pregnancy $(p=0.019)$ and during pregnancy $(p=0.036)$, with adequate weight gain. Obesity, on the other hand, was associated with excessive gain $(p=0.000)$. There was an association between gestational diabetes and obesity $(p=0.013)$, as well as between chronic arterial hypertension and obesity $(p=0.020)$. Most reported using electronic equipment simultaneously with meals and having consumed fresh foods the day before the interview, while the consumption of ultra-processed foods varied between $20 \%$ and $37 \%$. However, $68.5 \%$ reported having consumed sweetened drinks. Conclusion: This study found a higher prevalence of obesity before and during pregnancy, in addition to a significant percentage of consumption of fresh foods, and less frequent consumption of ultra-processed foods.
\end{abstract}

\section{DESCRIPTORS}

Nutritional Status. Food Consumption. Pregnant Women. Feeding Behavior. Food Guide. ${ }^{1}$ Nutricionista, Residente Multiprofissional em Neonatologia pela Secretaria Municipal de Saúde de São Paulo, do Hospital Municipal Mater-
nidade Escola Dr. Mário de Moraes Altenfelder Silva. São Paulo-SP, Brasil.

${ }^{2}$ Nutricionista, Mestre em Saúde Materno-Infantil, Docente do Curso de Nutrição da Universidade Santo Amaro. São Paulo-SP, Brasil. 
A s mudanças que ocorrem no organismo da mulher durante a gestação têm como finalidade criar um ambiente favorável ao crescimento e desenvolvimento do concepto. Ocorrem alterações no volume e composição sanguíneos, nas funções cardiovascular, pulmonar e renal. Além disso, ocorre alteração no sistema gastrointestinal que afeta o estado nutricional, devido à diminuição da ingestão de alimentos pelo possível surgimento de náuseas e vômitos, seguidos de retorno de apetite, muitas vezes excessivo. Somado a isso, pode ocorrer refluxo gástrico, em decorrência do relaxamento do esfíncter esofagiano inferior e pressão no estômago ocasionada pelo crescimento uterino ${ }^{1}$, impactando também no hábito alimentar.

Algumas gestações, por características específicas, podem implicar riscos tanto para a mãe quanto para o feto, apresentando maior probabilidade de desfechos desfavoráveis. Entre as condições que podem interferir na evolução normal da gestação estão as síndromes hipertensivas, o diabetes, e as anemias $^{2}$. Outro fator importante é a inadequação do ganho de peso, uma vez que o ganho insuficiente está associado à prematuridade e ao baixo peso ao nascer ${ }^{3}$, enquanto o ganho acima do recomendado está associado à hipertensão arterial, o diabetes e a macrossomia fetal ${ }^{2,4,5}$. Portanto, é imprescindível o monitoramento do estado nutricional, bem como do ganho ponderal durante a gestação.

Para indivíduos com idade entre 20 e 59 anos (exceto gestantes), a classificação do estado nutricional por meio do Índice de Massa Corporal (IMC) é realizada tendo como referência os pontos de corte preconizados pela World Health Organization (WHO), sendo classificados como baixo peso indivíduos com IMC $<18,5 \mathrm{Kg} / \mathrm{m}^{2}$, eutróficos $18,5-24,9 \mathrm{Kg} /$ $\mathrm{m}^{2}$, com sobrepeso $25,0-29,9 \mathrm{Kg} / \mathrm{m}^{2}$ e obesos $I M C \geq 30 \mathrm{Kg} / \mathrm{m}^{2}{ }^{6}$. O ganho de peso durante a gestação é determinado, preferencialmente, de acordo com o IMC pré-gestacional, classificado conforme os critérios da WHO. Com relação à avaliação do estado nutricional da gestante, preconiza-se que seja feita por meio do IMC, segundo a idade gestacional, utilizando-se a curva de Atalah ${ }^{7,8}$.

Estudos têm demonstrado uma alta prevalência de ganho de peso excessivo durante a gestação entre mulheres brasileiras ${ }^{9-12}$, resultado que tem sido relacionado ao IMC pré-gestacional e à alimentação inadequa$\mathrm{da}^{10,12}$. Embora seja importante o incremento na quantidade de calorias ingeridas, para manter as demandas metabólicas da gestação e do crescimento fetal ${ }^{1}$, é necessário que seja feito de maneira apropriada, atentando-se à qualidade da alimentação.

Com o objetivo de apoiar e incentivar as práticas alimentares saudáveis, o Ministério da Saúde publicou em 2014 o Guia Alimentar para a População Brasileira. Suas recomendações podem ser utilizadas para indivíduos sadios e enfermos, com adaptações às condições específicas, abrangendo todos os ciclos de vida. Recomenda-se um maior consumo de alimentos in natura e minimamente processados e a utilização de óleos, gorduras, sal e açúcar em pequenas quantidades. Sugere-se, ainda, o consumo limitado de alimentos processados e evitar os ultraprocessados. Além disso, o Guia chama a atenção para o comportamento alimentar, sugerindo comer com regularidade e atenção, em ambientes apropriados e, quando possível, em companhia ${ }^{13}$.

Os alimentos in natura são os obtidos 
diretamente de plantas ou de animais, e adquiridos para o consumo sem sofrer alterações após deixarem a natureza, como folhas, frutos, ovos e leite. Enquanto os minimamente processados são alimentos in natura que, antes de sua aquisição, sofreram alterações mínimas, como grãos secos, polidos e empacotados, ou moídos na forma de farinhas. São exemplos de alimentos processados as conservas de legumes, compotas de frutas, queijos e pães. Os ultraprocessados incluem biscoitos recheados, salgadinhos "de pacote", refrigerantes e macarrão "instantâneo"13.

Baseando-se nas recomendações do Guia, foram criados formulários para avaliação de marcadores de consumo alimentar, com objetivo de identificar práticas alimentares saudáveis e não saudáveis ${ }^{14}$. Tais instrumentos integram uma ação de Vigilância Alimentar e Nutricional (VAN), atendendo à terceira diretriz da Política Nacional de Alimentação e Nutrição (PNAN) ${ }^{14,15}$. Trata-se de uma proposta de avaliação rápida do consumo alimentar, para pessoas de qualquer idade ou fase do curso da vida, incluindo as gestantes ${ }^{14}$.

O objetivo do presente estudo foi descrever o estado nutricional e o consumo alimentar de pacientes internadas na Casa da Gestante de Alto Risco, de um Hospital e Maternidade no Município de São Paulo.

\section{METODOLOGIA}

Trata-se de um estudo transversal, descritivo, realizado na Casa da Gestante de Alto Risco (CGAR), de um Hospital e Maternidade da rede pública do Município de São Paulo. A CGAR possui 20 leitos e recebe gestantes com patologias ou complicações que necessitam de maior atenção, como o diabetes, a hipertensão, o trabalho de parto prematuro, dentre outras.

Foram convidadas a participar da pesquisa, as pacientes internadas na CGAR, independente da idade gestacional, com no máximo 1 (um) dia de internação, pois o presente trabalho avaliou o consumo alimentar de um dia habitual, desconsiderando as refeições realizadas após a internação. Foram excluídas da pesquisa as gestantes internadas há mais de um dia; impossibilitadas de deambular para aferição de peso e estatura; em condições clínicas que impossibilitaram responder ao questionário; com gestação gemelar; e com idade inferior a 18 anos. A amostra obtida foi de 54 gestantes.

A coleta de dados ocorreu entre os meses de maio e setembro de 2019, por meio de entrevista, utilizando o formulário de marcadores de consumo alimentar do Ministério da Saúde. Tal formulário possui questões relacionadas à frequência de realização de refeições, ao hábito de utilizar aparelhos eletrônicos no momento das refeições e ao consumo de alimentos ou grupos de alimentos específicos no dia anterior à entrevista. Além disso, foi questionado o peso anterior à gestação e a idade. Posteriormente, confrontou-se o peso informado pela participante com o registrado na carteira da gestante e, nos casos de divergência, optou-se por utilizar o dado contido na carteira da gestante, uma vez que foi coletado no início da gestação, tendo menor possibilidade de erro. Não existindo registro no cartão, considerava-se o peso relatado. Uma das participantes não soube informar o peso pré-gestacional, nem havia essa informação na carteira da gestante, além disso, o primeiro registro de peso foi com 20 semanas de gestação, portanto, não foi possível calcular 
o IMC pré-gestacional, nem o ganho de peso. Quanto ao diagnóstico clínico de internação das gestantes, foi considerado o registrado no prontuário.

No momento da entrevista, aferiu-se o peso e a estatura das participantes, utilizando uma balança digital, com régua antropométrica acoplada, da marca Líder ${ }^{\circledR}$, disponível na enfermaria. Em seguida, foi calculado o IMC pré-gestacional e o IMC gestacional, classificando o estado nutricional em baixo peso, eutrofia, sobrepeso e obesidade, usando para o IMC pré-gestacional os critérios estabelecidos pela $\mathrm{WHO}$ (baixo peso IMC $<18,5 \mathrm{Kg} / \mathrm{m}^{2}$, eutrofia $18,5-24,9 \mathrm{Kg} / \mathrm{m}^{2}$, sobrepeso $25,0-29,9 \mathrm{Kg} / \mathrm{m}^{2}$ e obesidade $\mathrm{IMC} \geq 30 \mathrm{Kg} / \mathrm{m}^{2}$ ) e para o IMC, no momento da entrevista, a curva de Atalah ${ }^{8}$, de acordo com a idade gestacional.

As variáveis coletadas foram: idade; diagnóstico clínico de internação; IMC pré-gestacional; IMC gestacional; costume de realizar as refeições assistindo TV, usando computador e/ou celular; refeições realizadas ao longo do dia (café da manhã, lanche da manhã, almoço, lanche da tarde, jantar e ceia); e consumo de alimentos específicos (feijão, frutas frescas, verduras e/ou legumes, hambúrguer e/ou embutidos, bebidas adoçadas, macarrão instantâneo, salgadinhos de pacote ou biscoitos salgados, biscoito recheado, doces ou guloseimas).

Também foi calculado o ganho de peso gestacional até o momento da entrevista, bem como a adequação do ganho ponderal, levando em consideração as recomendações de ganho de peso contidas no Caderno de Atenção Básica $n^{\circ} 32$ (sobre a atenção ao pré-natal) e do manual de Orientações para a Coleta e Análise de Dados Antropométricos em Serviços de Saúde, ambos do Ministério da Saúde. Tais documentos preconizam que as mulheres que iniciam a gestação com baixo peso devem ganhar, em média, 2,3Kg no primeiro trimestre e $0,5 \mathrm{Kg}$ por semana nos semestres seguintes, as eutróficas devem ganhar $1,6 \mathrm{Kg}$ no primeiro trimestre e $0,4 \mathrm{Kg}$ por semana nos semestres seguintes, enquanto as com sobrepeso podem ganhar até $0,9 \mathrm{Kg}$ no primeiro trimestre e $0,3 \mathrm{Kg}$ por semana nos semestres seguintes, já as com obesidade, não precisam ganhar peso no primeiro trimestre e recomenda-se o ganho de $0,2 \mathrm{Kg}$ por semana no segundo e terceiro trimestres ${ }^{7,16}$. Considerou-se também a divisão dos trimestres gestacionais contida na Carteira da Gestante do Ministério da Saúde: primeiro trimestre até a $13^{a}$ semana; segundo da $14^{a}$ a $26^{a}$; e terceiro a partir da $27^{a 17}$. Tendo isso em vista, foram desenvolvidas fórmulas para verificar a adequação do ganho ponderal até o momento da entrevista, considerando a classificação do estado nutricional pré-gestacional, uma vez que a previsão do ganho de peso durante a gestação é feita, preferencialmente, de acordo com o IMC pré-gestacional. Sendo assim, as fórmulas utilizadas foram: (((idade gestacional atual - 13 semanas) $\times 0,5 \mathrm{Kg})+2,3 \mathrm{Kg}$ ) para baixo peso; (((idade gestacional atual - 13 semanas) $\times 0,4 \mathrm{Kg})+1,6 \mathrm{Kg}$ ) para eutrofia; (((idade gestacional atual -13 semanas) $x$ $0,3 \mathrm{Kg})+0,9 \mathrm{Kg}$ ) para sobrepeso; e ((idade gestacional atual - 13 semanas) $\times 0,2 \mathrm{Kg}$ ) para obesidade. Comparou-se então o ganho de peso até o momento da entrevista (peso pré-gestacional - peso atual) com o ganho previsto, calculado por meio das fórmulas. Por fim, o ganho ponderal foi classificado em insuficiente, adequado e excessivo.

Os dados da pesquisa foram tabulados em planilha do Microsoft Excel, e posterior- 
mente importados para o programa estatístico Stata versão 11.2. Para as associações estatísticas utilizou-se o teste do Qui-quadrado de Pearson e o teste exato de Fisher, considerando estatisticamente significante um valor de $p \leq 0,05$.

O projeto foi aprovado pelo Comitê de Ética em Pesquisa (CEP) da instituição proponente, Hospital Municipal e Maternidade Escola Dr. Mário de Moraes Altenfelder Silva (CAAE: 12287219.1.00005454), bem como da Universidade Santo Amaro (UNISA), instituição coparticipante. As participantes assinaram o Termo de Consentimento Livre e Esclarecido (TCLE).

\section{RESULTADOS}

Observou-se maior percentual de gestantes na faixa de idade superior a 30 anos, principalmente acima dos 35 (33,3\%) (Tabela 1). A maioria estava no terceiro trimestre gestacional no momento da entrevista $(83,3 \%)$ e o diagnóstico de internação mais prevalente foi o diabetes gestacional com $42,6 \%$, seguido de hipertensão arterial crônica com 20,4\%. Diagnósticos com $\mathrm{n}=1$ não foram considerados. Com relação ao estado nutricional, tanto no pré-gestacional quanto no gestacional, existiu maior prevalência de sobrepeso e obesidade. Comparando os dois períodos, observou-se diminuição no percentual de mulheres com baixo peso, caindo de $3,8 \%(n=2)$ para $1,9 \%$ $(n=1)$. O mesmo aconteceu com o percentual de mulheres com sobrepeso, diminuindo de $32,1 \%(n=17)$ para $22,2 \%(n=12)$. Em contrapartida, houve um aumento no percentual de eutrofia e obesidade durante a gestação, subindo de $22,6 \%(n=12)$ para $25,9 \%(n=14)$, e de $41,4 \%(n=22)$ para $50 \%(n=27)$, respectivamente.

Existiu uma associação estatisticamente significante entre a eutrofia e o ganho de peso adequado, tanto com o estado nutricional pré-gestacional $(p=0,019)$ quanto com o gestacional $(p=0,036)$., além de uma associação inversa entre a eutrofia e o ganho de peso excessivo $(p=0,003)$. Por outro lado, a obesidade mostrou-se diretamente associada ao ganho excessivo $(p=0,000)$ e inversamente associada ao ganho abaixo da recomendação $(p=0,008)$ (Tabela 2).

Foi possível observar uma associação inversa entre o diabetes gestacional e a eutrofia $(p=0,013)$ e direta entre o diabetes gestacional e a obesidade ( $p=0,013)$, assim como entre a hipertensão arterial crônica e a obesidade $(p=0,020)$ (Tabela 3$)$. Das 6 gestantes com diagnóstico de restrição de crescimento intrauterino, 4 foram classificadas como eutróficas no momento da entrevista $(p=0,033)$. Resultado semelhante foi observado entre as participantes com oligoâmnio, sendo que, das 4 com esse diagnóstico, 3 estavam eutróficas no momento da entrevista $(p=0,049)$. As 2 gestantes em trabalho de parto prematuro apresentaram sobrepeso $(p=0,046)$.

Quanto à associação entre o ganho de peso gestacional e o diagnóstico de internação, apenas existiu significância estatística entre a hipertensão arterial crônica e o ganho excessivo, com valor de $p=0,016$ (Tabela 4).

A maioria das gestantes referiu realizar as refeições principais (café da manhã, almoço e jantar) e o lanche da tarde (Tabela 5). Além disso, nota-se que a maioria relatou o uso de equipamentos eletrônicos, simultaneamente, à realização das refeições $(70,4 \%)$. No que se refere ao consumo alimentar, um expressivo 
Tabela 1. Caracterização das gestantes entrevistadas segundo idade em anos, trimestre gestacional, estado nutricional pré-gestacional e gestacional, e diagnóstico de internação. São Paulo-SP, 2019.

\begin{tabular}{|c|c|c|}
\hline Variáveis & $\mathrm{n}$ & $\%$ \\
\hline \multicolumn{3}{|l|}{ Idade (anos) } \\
\hline$<20$ & 03 & 5,6 \\
\hline$\geq 20<25$ & 09 & 16,7 \\
\hline$\geq 25<30$ & 08 & 14,8 \\
\hline$\geq 30<35$ & 16 & 29,6 \\
\hline$\geq 35$ & 18 & 33,3 \\
\hline \multicolumn{3}{|l|}{ Idade gestacional } \\
\hline $1^{\circ}$ trimestre & 02 & 3,7 \\
\hline $2^{\circ}$ trimestre & 07 & 13,0 \\
\hline $3^{\circ}$ trimestre & 45 & 83,3 \\
\hline \multicolumn{3}{|l|}{ Estado nutricional pré-gestacional ${ }^{*}$} \\
\hline Baixo peso & 02 & 3,8 \\
\hline Eutrofia & 12 & 22,6 \\
\hline Sobrepeso & 17 & 32,1 \\
\hline Obesidade & 22 & 41,5 \\
\hline \multicolumn{3}{|l|}{ Estado nutricional gestacional } \\
\hline Baixo peso & 01 & 1,9 \\
\hline Eutrofia & 14 & 25,9 \\
\hline Sobrepeso & 12 & 22,2 \\
\hline Obesidade & 27 & 50,0 \\
\hline \multicolumn{3}{|l|}{ Diagnóstico de internação } \\
\hline Diabetes do tipo 1 (DM1) & 02 & 3,7 \\
\hline Diabetes do tipo 2 (DM2) & 05 & 9,3 \\
\hline Diabetes gestacional (DMG) & 23 & 42,6 \\
\hline Doença hipertensiva específica da gestação (DHEG) & 05 & 9,3 \\
\hline Hipertensão arterial crônica (HAC) & 11 & 20,4 \\
\hline Restrição de crescimento intrauterino (RCIU) & 06 & 11,1 \\
\hline Oligoâmnio & 04 & 7,4 \\
\hline Hipotireoidismo & 02 & 3,7 \\
\hline Pré-eclâmpsia & 07 & 13,0 \\
\hline Pielonefrite & 04 & 7,4 \\
\hline Trabalho de parto prematuro (TPP) & 02 & 3,7 \\
\hline
\end{tabular}

percentual informou ter consumido alimentos in natura ou minimamente processados no dia anterior à entrevista: feijão $(72,2 \%)$, frutas $(70,4 \%)$, verduras e legumes $(70,2 \%)$. Em contrapartida, os alimentos ultraprocessados foram menos relatados, com percentuais variando entre $20 \%$ e $37 \%$. No entanto, $68,5 \%$ referiram ter consumido bebidas adoçadas.

\section{DISCUSSÃO}

No presente estudo, observou-se um percentual elevado de mulheres com mais de 35 anos, possivelmente, por a amostra ter sido composta por gestantes internadas em enfermaria de alto risco. Nessa faixa etária, a gestação oferece mais riscos tanto pela 
Tabela 2. Associação do estado nutricional pré-gestacional e gestacional com o ganho de peso na gestação. São Paulo-SP, 2019.

\begin{tabular}{|c|c|c|c|c|c|c|c|c|c|}
\hline \multirow{3}{*}{ Variáveis } & \multicolumn{9}{|c|}{ Ganho de peso gestacional } \\
\hline & \multicolumn{3}{|c|}{$\begin{array}{l}\text { Baixo } \\
(n=21)\end{array}$} & \multicolumn{3}{|c|}{$\begin{array}{l}\text { Adequado } \\
(n=6)\end{array}$} & \multicolumn{3}{|c|}{ Excessivo $(n=26)$} \\
\hline & Sim & Não & $p$ & Sim & Não & $p$ & Sim & Não & $p$ \\
\hline \multicolumn{10}{|c|}{$\begin{array}{l}\text { Estado nutricional pré- } \\
\text { gestacional }{ }^{*}\end{array}$} \\
\hline Baixo peso $(n=2)$ & 01 & 20 & 0,640 & - & 06 & 0,784 & 01 & 25 & 0,745 \\
\hline Eutrofia $(n=12)$ & 04 & 17 & 0,438 & 04 & 02 & 0,019 & 04 & 22 & 0,182 \\
\hline Sobrepeso $(n=17)$ & 09 & 12 & 0,173 & 01 & 05 & 0,364 & 07 & 19 & 0,430 \\
\hline Obesidade $(n=22)$ & 07 & 14 & 0,328 & 01 & 05 & 0,195 & 14 & 12 & 0,074 \\
\hline \multicolumn{10}{|l|}{$\begin{array}{l}\text { Estado nutricional } \\
\text { gestacional }\end{array}$} \\
\hline Baixo peso $(n=1)$ & 01 & 20 & 0,396 & - & 06 & 0,887 & - & 26 & 0,509 \\
\hline Eutrofia $(n=14)$ & 08 & 13 & 0,118 & 04 & 02 & 0,036 & 02 & 24 & 0,003 \\
\hline Sobrepeso $(n=11)$ & 06 & 15 & 0,214 & 01 & 05 & 0,636 & 04 & 22 & 0,273 \\
\hline Obesidade $(n=27)$ & 06 & 15 & 0,008 & 01 & 05 & 0,087 & 20 & 06 & 0,000 \\
\hline
\end{tabular}

Tabela 3. Associação do estado nutricional gestacional com o diagnóstico de internação. São Paulo-SP, 2019.

\begin{tabular}{|c|c|c|c|c|c|c|c|c|c|c|c|c|}
\hline \multirow{2}{*}{ Diagnóstico } & \multicolumn{4}{|c|}{ Baixo peso $(n=1)$} & \multicolumn{2}{|c|}{$\begin{array}{c}\text { Eutrofia } \\
(n=14)\end{array}$} & \multicolumn{3}{|c|}{ Sobrepeso $(n=12)$} & \multicolumn{3}{|c|}{ Obesidade $(n=27)$} \\
\hline & Sim & Não & $p$ & Sim & Não & $p$ & Sim & Não & $p$ & Sim & Não & $p$ \\
\hline DM1 $(n=2)$ & - & 01 & 0,963 & 01 & 13 & 0,455 & 01 & 11 & 0,398 & - & 27 & 0,245 \\
\hline $\mathrm{DM} 2(\mathrm{n}=5)$ & - & 01 & 0,907 & 01 & 13 & 0,613 & - & 12 & 0,269 & 04 & 23 & 0,175 \\
\hline DMG $(n=23)$ & - & 01 & 0,574 & 02 & 12 & 0,013 & 05 & 07 & 0,941 & 16 & 11 & 0,013 \\
\hline DHEG $(n=5)$ & - & 01 & 0,907 & - & 14 & 0,208 & 01 & 11 & 0,694 & 04 & 23 & 0,175 \\
\hline $\mathrm{HAC}(n=11)$ & - & 01 & 0,796 & 01 & 13 & 0,148 & 01 & 11 & 0,229 & 09 & 18 & 0,020 \\
\hline $\operatorname{RCIU}(n=6)$ & - & 01 & 0,889 & 04 & 10 & 0,033 & 01 & 11 & 0,598 & 01 & 26 & 0,096 \\
\hline Oligoâmnio (n & - & 01 & 0,926 & 03 & 11 & 0,049 & - & 12 & 0,354 & 01 & 26 & 0,305 \\
\hline Hipotireoidismo $(n=2)$ & - & 01 & 0,963 & 01 & 13 & 0,455 & - & 12 & 0,602 & 01 & 26 & 0,755 \\
\hline Pré-eclâmpsia $(n=7)$ & - & 01 & 0,870 & 02 & 12 & 0,591 & 02 & 10 & 0,492 & 03 & 24 & 0,500 \\
\hline Pielonefrite $(n=4)$ & - & 01 & 0,926 & 01 & 13 & 0,726 & 01 & 11 & 0,646 & 02 & 25 & 0,695 \\
\hline $\operatorname{TPP}(n=2)$ & - & 01 & 0,963 & - & 14 & 0,545 & 02 & 10 & 0,046 & - & 27 & 0,245 \\
\hline
\end{tabular}


Tabela 4. Associação do ganho de peso gestacional com o diagnóstico de internação. São Paulo-SP, 2019.

\begin{tabular}{|c|c|c|c|c|c|c|c|c|c|}
\hline \multirow{3}{*}{ Diagnóstico } & \multicolumn{9}{|c|}{ Ganho de peso gestacional ${ }^{*}$} \\
\hline & \multicolumn{3}{|c|}{$\begin{array}{l}\text { Baixo } \\
(n=21)\end{array}$} & \multicolumn{3}{|c|}{$\begin{array}{c}\text { Adequado } \\
(n=6)\end{array}$} & \multicolumn{3}{|c|}{ Excessivo $(n=26)$} \\
\hline & Sim & Não & $p$ & Sim & Não & $p$ & Sim & Não & $\mathrm{p}$ \\
\hline $\mathrm{DM} 1(\mathrm{n}=2)$ & 0 & 21 & 0,360 & 0 & 6 & 0,784 & 2 & 24 & 0,236 \\
\hline $\mathrm{DM} 2(\mathrm{n}=5)$ & 1 & 20 & 0,333 & 0 & 6 & 0,535 & 4 & 22 & 0,164 \\
\hline DMG $(n=23)$ & 8 & 13 & 0,528 & 2 & 4 & 0,471 & 13 & 13 & 0,341 \\
\hline DHEG $(n=5)$ & 2 & 19 & 0,667 & 0 & 6 & 0,535 & 3 & 23 & 0,482 \\
\hline $\operatorname{HAC}(n=11)$ & 2 & 19 & 0,097 & 0 & 6 & 0,229 & 9 & 17 & 0,016 \\
\hline $\operatorname{RCIU}(n=6)$ & 4 & 17 & 0,160 & 1 & 5 & 0,532 & 1 & 25 & 0,104 \\
\hline Oligoâmnio (n=4) & 3 & 18 & 0,166 & 0 & 6 & 0,609 & 1 & 25 & 0,320 \\
\hline Hipotireoidismo $(n=2)$ & 1 & 20 & 0,640 & 0 & 6 & 0,784 & 1 & 25 & 0,745 \\
\hline Pré-eclâmpsia (n=7) & 2 & 19 & 0,420 & 1 & 5 & 0,592 & 4 & 22 & 0,478 \\
\hline Pielonefrite $(n=4)$ & 1 & 20 & 0,656 & 0 & 6 & 0,692 & 2 & 24 & 0,486 \\
\hline $\operatorname{TPP}(n=2)$ & 1 & 20 & 0,640 & 1 & 5 & 0,216 & 0 & 26 & 0,255 \\
\hline
\end{tabular}

Tabela 5. Frequência da realização de refeições, do uso de aparelhos eletrônicos e de alimentos consumidos pelas gestantes entrevistadas. São Paulo-SP, 2019.

\begin{tabular}{lll}
\hline \multicolumn{1}{c}{ Variáveis } & $\mathrm{n}$ & $\%$ \\
\hline Frequência da realização de refeições & & \\
Café da manhã & 46 & 85,2 \\
Lanche da manhã & 20 & 37,0 \\
Almoço & 51 & 94,4 \\
Lanche da tarde & 44 & 81,5 \\
Jantar & 52 & 96,3 \\
Ceia & 25 & 46,3 \\
Alimentos consumidos & & \\
Feijão & 39 & 72,2 \\
Frutas frescas & 38 & 70,4 \\
Verduras e/ou legumes & 39 & 72,2 \\
Hambúrguer e/ou embutidos & 18 & 33,3 \\
Bebidas adoçadas & 37 & 68,5 \\
Macarrão instantâneo, salgadinhos de pacote ou biscoitos salgados & 11 & 20,4 \\
Biscoito recheado, doces ou guloseimas & 20 & 37,0 \\
Uso de aparelhos eletrônicos & 38 & 70,4 \\
\hline
\end{tabular}


maior possibilidade de desfechos obstétricos desfavoráveis, quanto por doenças crônicas preexistentes ${ }^{18}$. Além disso, a maioria estava no terceiro trimestre de gestação, momento no qual ocorre o agravamento de condições preexistentes, como por exemplo, a intolerância à glicose ${ }^{19}$, fato observado no presente trabalho, uma vez que o diagnóstico mais prevalente foi o diabetes gestacional.

Quanto ao estado nutricional pré-gestacional, ocorreu uma maior prevalência de obesidade, seguido de sobrepeso e eutrofia. Foi observada a mesma tendência para o IMC gestacional, com aumento na prevalência de obesidade e eutrofia, provavelmente, devido à migração de parte das gestantes das categorias de baixo peso e sobrepeso, pois existiu uma diminuição na prevalência destas últimas. Resultado semelhante foi encontrado em estudo realizado no município de Candelária-RS, no qual $50,9 \%$ das participantes apresentaram excesso de peso (sobrepeso e obesidade), já no período gestacional, o resultado foi o inverso do obtido no presente trabalho, pois as autoras evidenciaram um incremento no baixo peso e na redução da eutrofia e do excesso de peso $^{3}$. Enquanto outro trabalho, também com gestantes de alto risco no Município de Maceió, verificou que $19,7 \%$ das mulheres tinham baixo peso e $48,3 \%$ apresentavam excesso de peso durante a gestação ${ }^{20}$. Outros estudos também encontraram maior prevalência de sobrepeso e de obesidade antes da gestação, com incremento no período gestacional ${ }^{21,22}$.

Em contrapartida, Nunes (2015) em pesquisa realizada na Região Sudeste, observou que $61,04 \%$ das mulheres iniciaram a gestação com o IMC considerado adequado, porém no terceiro trimestre gestacional, $61,1 \%$ apresentavam sobrepeso e $15 \%$ estavam obesas $^{9}$. Silva et al. (2018) bem como Fraga et al. (2014) encontraram uma prevalência de IMC pré-gestacional adequado de $66,3 \%$ e $54,1 \%$, respectivamente ${ }^{4,23}$. No entanto, estes dois últimos estudos foram realizados com gestantes atendidas na Atenção Primária.

Ocorreu uma prevalência expressiva de ganho de peso fora do recomendado durante a gestação, pois $39,6 \%(n=21)$ das gestantes ganharam peso de forma insuficiente, 49,05\% ( $n=26)$ ganharam de forma excessiva e, apenas, $11,3 \%(n=6)$ ganharam dentro do recomendado. Encontra-se bem estabelecida na literatura a relação entre o ganho de peso excessivo e os desfechos adversos na gestação, como a macrossomia fetal ${ }^{2}$, porém, no presente estudo, também chama atenção a prevalência de ganho ponderal insuficiente, sendo que, das 21 gestantes nesta condição, $19,04 \%(n=4)$ iniciaram a gestação eutróficas, $42,85 \%(n=9)$ com sobrepeso, e 33,33\% ( $n=7)$ com obesidade, ou seja, mais de $70 \%$ das que ganharam peso de forma insuficiente apresentavam excesso de peso antes de engravidar, sugerindo a existência de maior preocupação com o ganho ponderal entre as mulheres com IMC nesta classificação. Embora o resultado não tenha sido estatisticamente significante, é importante ressaltar que a perda de peso ou o ganho insuficiente durante a gestação, independente do IMC pré-gestacional, também pode trazer riscos, como a prematuridade e o baixo peso ao nascer ${ }^{3,20}$.

Com relação à associação entre o estado nutricional e o ganho de peso gestacional, em estudo realizado em Pará de Minas-MG, as autoras também encontraram maior predominância de ganho de peso adequado entre as mulheres eutróficas, porém, ganho excessivo entre as com sobrepeso, obser- 
vando também maior número de gestantes com ganho de peso acima do ideal $(37,5 \%)$, seguido de ganho insuficiente $(32,8 \%)$ e ganho adequado $(29,7 \%)$, corroborando com os achados do presente estudo ${ }^{24}$. Nunes (2015) e Carvalhaes et al. (2013) também encontraram associação entre o estado nutricional e o ganho de peso gestacional ${ }^{9,12}$.

O diabetes gestacional e a hipertensão arterial crônica têm como um dos fatores de risco o excesso de peso ${ }^{2}$. Isso justifica a associação encontrada, no presente estudo, entre o diabetes gestacional e a obesidade e entre a hipertensão arterial crônica com a obesidade e o ganho excessivo de peso, indo de encontro ao relatado na literatura ${ }^{21,23}$. Ambas as comorbidades estão entre as principais causas de morbimortalidade materna e fetal ${ }^{25,26}$. Por outro lado, o ganho de peso insuficiente é uma possível explicação para a associação encontrada entre os diagnósticos de restrição de crescimento intrauterino e oligoâmnio, com estado nutricional gestacional de eutrofia ${ }^{27,28}$, uma vez que, das 14 gestantes classificadas como eutróficas, $57,14 \%(n=8)$ apresentaram ganho ponderal abaixo do recomendado.

A alimentação e a nutrição são fatores determinantes e condicionantes da saúde. Por este motivo, a Vigilância Alimentar e Nutricional (VAN) tem como um dos pilares o monitoramento das práticas de consumo alimentar ${ }^{14}$. A inadequação do estado nutricional materno pode comprometer o crescimento fetal, por isso, os hábitos alimentares saudáveis são fundamentais para um bom prognóstico da gestação ${ }^{7}$. O presente estudo encontrou prevalências acima de $85 \%$ de realização das refeições principais e de $37 \%$ a $81 \%$ de pequenas refeições. Silva et al. (2018), em estudo com 95 gestantes, verificaram que $51,6 \%$ delas costumavam fazer 3 refeições por dia e mais três lanches saudáveis ${ }^{23}$.

O Guia Alimentar para a População Brasileira incentiva o comer com atenção, sugerindo comer devagar e sem se envolver em outras atividades, evitando o uso de telefone celular e da televisão ${ }^{13}$. A prevalência do uso de aparelhos eletrônicos, simultaneamente, à realização das refeições foi alta neste estudo $(70,4 \%)$, fato preocupante, uma vez que a distração pode levar à ingestão excessiva de alimentos.

Outra recomendação do Guia é preferir sempre alimentos in natura ou minimamente processados e preparações culinárias, a alimentos ultraprocessados. As razões para evitar o consumo de ultraprocessados vão desde a inadequação nutricional destes produtos, ao impacto que suas formas de produção, distribuição, comercialização e consumo têm sobre a cultura, a vida social e sobre o meio ambiente. São alimentos desbalanceados nutricionalmente, frequentemente ricos em sódio, gorduras e/ou açúcar, além de serem pobres em fibras, vitaminas, minerais e outras sustâncias com atividade biológica. O consumo frequente destes produtos favorece o desenvolvimento de doenças como o diabetes, a hipertensão, alguns tipos de câncer e as doenças cardiovasculares. Por outro lado, os alimentos in natura ou minimamente processados, são a base para uma alimentação balanceada, que respeita a cultura, além de promover um sistema alimentar socialmente e ambientalmente sustentável ${ }^{13}$.

No presente estudo, o consumo de alimentos in natura ou minimamente processados no dia anterior à entrevista foi referido por aproximadamente $70 \%$ das gestantes. En- 
quanto, o consumo de ultraprocessados, variou entre $20 \%$ e $37 \%$. Crivellentil et al. (2018) em trabalho com objetivo de desenvolver um índice de qualidade da dieta adaptado para gestantes, observaram que $67,9 \%$ das gestantes atingiram a pontuação máxima para o consumo de hortaliças, $90,3 \%$ para leguminosas, $18,3 \%$ para frutas frescas, porém apenas $15,3 \%$ consumiram alimentos ultraprocessados, dentro do limite estabelecido ${ }^{29}$. Enquanto, Silva et al. (2018) constataram que $52,6 \%$ das gestantes entrevistadas consumiam alimentos naturais todos os dias ${ }^{23}$.

O expressivo percentual de consumo de alimentos in natura ou minimamente processados e o menor percentual de alimentos ultraprocessados encontrado neste estudo, pode ser explicado pelo fato da amostra ter sido composta, majoritariamente, por gestantes com patologias que requerem mudanças nos hábitos alimentares, como o diabetes e a hipertensão arterial, havendo a possibilidade de terem recebido orientação nutricional em algum momento do pré-natal.

O consumo frequente de bebidas adoçadas também contribui para o aumento do risco de obesidade, devido a menor capacidade de registro pelo organismo das calorias provenientes destes produtos ${ }^{13} .68,5 \%$ das gestantes referiram ter consumido bebidas adoçadas no dia anterior à entrevista, chamando atenção para a importância de investigar a frequência deste consumo e orientar sobre os riscos durante o acompanhamento nos serviços de saúde.

Entre as limitações deste estudo, é possível citar o tamanho da amostra, tendo em vista que uma amostra maior poderia contribuir com o aumento das associações, com significância estatística. Outra limitação, relaciona-se à coleta dos dados de consumo alimentar, pois as informações são apenas de um dia, não sendo possível inferir se representa o consumo habitual. Além disso, não foram coletadas informações sobre a quantidade ingerida, em virtude do tipo de formulário escolhido, pois o mesmo propõe-se a ser uma avaliação rápida do consumo alimentar.

\section{CONCLUSÃO}

O presente estudo encontrou maior prevalência de excesso de peso tanto no período pré-gestacional quanto durante a gestação. Observou-se também alta prevalência de ganho ponderal inadequado (excessivo e insuficiente). Em contrapartida, evidenciou-se um expressivo percentual de consumo de alimentos in natura ou minimamente processados e menor frequência do consumo de ultraprocessados. No entanto, hábitos como o uso de aparelhos eletrônicos, simultaneamente, à realização das refeições e a ingestão de bebidas adoçadas requerem atenção, mostrando a necessidade da elaboração de estratégias mais efetivas de orientação sobre as práticas alimentares e os hábitos saudáveis, que alcancem mulheres em idade reprodutiva, gestantes, bem como toda a comunidade usuária dos serviços de saúde. Por fim, sugere-se a realização de mais estudos que investiguem o consumo alimentar, utilizando a classificação dos alimentos pelo grau de processamento, assim como, o comportamento alimentar e que considerem as questões socioeconômicas e o acesso à alimentação saudável, por se tratarem de fatores que contribuem substancialmente para a determinação das práticas alimentares. 


\section{REFERÊNCIAS}

1. Mahan LK, Scott-Stump S, Raymond JL. Krause: Alimentos, Nutrição e Dietoterapia. 13. ed. Rio de Janeiro: Elsevier, 2012.

2. Ministério da Saúde $(B R)$, Secretaria de Atenção à Saúde. Gestação de Alto Risco: manual técnico. 5. ed. Brasília (DF); 2010.

3. Pereira VR, Wichmann FMA. Estado nutricional materno e peso ao nascer do bebê no município de Candelária-RS. Cinergis. 2016; 17(4Supl.1):368-372.

4. Fraga ACSA, Theme Filha MM. Factors associated with gestational weight gain in pregnant women in Rio de Janeiro, Brazil, 2008. Cad Saúde Pública. 2014; 30(3):633-644.

5. Nogueira AI, Carreiro MP. Obesidade e gravidez. Rev. Med. Minas Gerais. 2013; 23(1):88-98.

6. World Health Organization [internet]. Geneva; c2018.

7. Ministério da Saúde (BR), Secretaria de Atenção à Saúde. Atenção ao pré-natal de baixo risco. Brasília (DF); 2012.

8. Atalah SE, Castillo LC, Castro SR, Aldea A. Propuesta de unnuevoestándar de evaluación nutricional enembarazadas. Rev Med Chil. 1997; 125(12):1429-1436.

9. Nunes CTG. Análise do ganho de peso gestacional em muIheres da região Sudeste do Brasil e desfechos perinatais [dissertação]. Faculdade de Saúde Pública da USP; 2015.

10. Magalhães EIS, Maia DS, Bonfim CFA, Netto MP, Lamounier JA, Rocha DS. Prevalência e fatores associados ao ganho de peso gestacional excessivo em unidades de saúde do sudoeste da Bahia. Rev Bras Epidemiol. 2015; 18(4):858-869.

11. Starling AP, Brinton JT, Glueck DH, Shapiro AL, Harrod CS, Lynch AM, et al. Associations of maternal BMI and gestational weight gain with neonatal adiposity in the Healthy Start study. Am J Clin Nutr. 2015; 101:302-9.

12. Carvalhaes MABL, Gomes CB, Malta MB, Papini SJ, Parada CMGL. Sobrepeso pré-gestacional associa-se a ganho ponderal excessivo na gestação. Rev Bras Ginecol Obstet. 2013; 35(11):523-9.

13. Ministério da Saúde (BR), Secretaria de Atenção à Saúde. Guia Alimentar para a População Brasileira. 2. ed. Brasília (DF); 2014.

14. Ministério da Saúde (BR), Secretaria de Atenção à Saúde. Orientações para Avaliação de Marcadores de Consumo Alimentar na Atenção Básica. Brasília (DF); 2015.

15. Ministério da Saúde (BR), Secretaria de Atenção à Saúde. Política Nacional de Alimentação e Nutrição. Brasília (DF); 2013.

16. Ministério da Saúde (BR), Secretaria de Atenção à Saúde. Orientações para a Coleta e Análise de Dados Antropométricos em Serviços de Saúde: Norma Técnica do Sistema de Vigilância Alimentar e Nutricional - SISVAN. Brasília (DF); 2011.
17. Ministério da Saúde (BR), Secretaria de Atenção à Saúde. Caderneta da Gestante. 4. ed. Brasília (DF); 2018.

18. Bezerra AC, Mesquita JS, Brito MCC, Santos RB, Teixeira FV. Desafios Enfrentados por Mulheres Primigestas em Idade Avançada. Rev Bras Ci Saúde. 2015; 19(2):163-168.

19. Muniz NA, Reis LBS. Terapia nutricional do Diabetes Mellitus na gestação. Com. Ciências Saúde. 2013; 24(4):363-374.

20. Oliveira ACM, Pereira LA, Ferreira RC, Clemente APG. Estado nutricional materno e sua associação com o peso ao nascer em gestações de alto risco. Ciênc. Saúde Coletiva. 2018; 23(7):2373-2382.

21. Manera F, Höfelmann DA. Excesso de peso em gestantes acompanhadas em unidades de saúde de Colombo, Paraná, Brasil. Demetra. 2019; v.14:e36842.

22. Santana AC. Consumo alimentar na gestação e ganho ponderal: um estudo de coorte de gestantes da zona oeste do município de São Paulo [dissertação]. Faculdade de Saúde Pública da USP; 2013.

23. Silva MG, Holanda VR, Lima LSV, Melo GP. Estado Nutriciona e Hábitos Alimentares de Gestantes Atendidas na Atenção Primária de Saúde. Rev Bras Ci Saúde. 2018; 22(4):349-356.

24. Eleutério BM, Araújo GLO, Silveira LP, Anastácio LR. Perfil nutricional materno e estado nutricional neonatal, na cidade de Pará de Minas - MG. Rev. Med. Minas Gerais 2013; 23(3):311-317.

25. Moura MDR, Castro MP, Margoto PR, Rugolo LMSS. Hipertensão Arterial na Gestação - importância do seguimento materno no desfecho neonatal. Com Ciências Saúde. 2011; 1(22Supl):S113-S120.

26. Sousa VBG, Meireles AVP, Frota JT, Garcia MMCM, Nobre RG. Gestação e Diabetes: Relação Entre Estado Nutricional e o Controle Glicêmico. Rev Bras Promoç Saúde. 2014; 27(4):541-549.

27. Neto ARM, Córdoba JCM, Peraçoli JC. Etiologia da restrição de crescimento intrauterino (RCIU). Com Ciências Saúde. 2011; 1 (22 supl):S21-S30.

28. Patriota AF, Guerra GVQL, Melo BCP, Santos AC, Torres Júnior, AC, Souza ASR. Volume de líquido amniótico e os desfechos maternos em gestantes com ruptura prematura das membranas pré-termo. Rev Bras Ginecol Obstet. 2014; 36(4):146-51.

29. Crivellenti LC, Zuccolotto DCC, Sartorelli DS. Desenvolvimento de um Índice de Qualidade da Dieta Adaptado para Gestantes. Rev. Saúde Pública. 2018; 52:59.

\section{CORRESPONDÊNCIA}

Rouglana Ribeiro Barbosa

Rua Iracema Sena Cerqueira Santos, 656

Cidade Intercap, Taboão da Serra-SP

CEP: 06757-240

E-mail: rouglana@hotmail.com 